\title{
BX open Gynaecological cancer follow-up: national survey of current practice in the UK
}

\author{
Simon Leeson, ${ }^{1}$ Nick Stuart, ${ }^{2}$ Yvonne Sylvestre, ${ }^{3}$ Liz Hall, ${ }^{1}$ Rhiannon Whitaker ${ }^{3}$
}

To cite: Leeson S, Stuart N, Sylvestre $\mathrm{Y}$, et al.

Gynaecological cancer followup: national survey of current practice in the UK. BMJ Open 2013;3:e002859.

doi:10.1136/bmjopen-2013002859

- Prepublication history and additional material for this paper is available online. To view these files please visit the journal online (http://dx.doi.org/10.1136/ bmjopen-2013-002859).

Received 9 March 2013 Revised 8 June 2013 Accepted 14 June 2013

${ }^{1}$ Department of Obstetrics and Gynaecology, Betsi Cadwaladr University Health Board, Bangor, Gwynedd, UK ${ }^{2}$ School of Medical Sciences, Bangor University, Bangor, Gwynedd, UK

${ }^{3}$ North Wales Organisation for Randomised Trials in Health, Bangor University, Bangor, Gwynedd, UK

Correspondence to Simon Leeson; simon.leeson@wales.nhs.uk

\section{ABSTRACT}

Objective: To establish a baseline of national practice for follow-up after treatment for gynaecological cancer.

Design: Questionnaire survey.

Setting: Gynaecological cancer centres and units.

Geographical location: UK.

Participants: Members of the British Gynaecological Cancer Society and the National Forum of

Gynaecological Oncology Nurses.

Interventions: A questionnaire survey.

Outcome measures: To determine schedules of follow-up, who provides it and what routine testing is used for patients who have had previous gynaecological cancer.

Results: A total of 117 responses were obtained; $115(98 \%)$ reported hospital scheduled regular followup appointments. Two involved general practitioners. Follow-up was augmented or replaced by telephone follow-up in 29 responses (25\%) and patient-initiated appointments in 38 responses (32\%). A total of $80(68 \%)$ cancer specialists also offered combined follow-up clinics with other specialties. Clinical examinations for hospital-based follow-up were mainly performed by doctors $(67 \%$ for scheduled regular appointments and $63 \%$ for patient-initiated appointments) while telephone follow-up was provided in the majority by nurses (76\%). Most respondents (76/ $117(65 \%))$ provided routine tests, of which 66/76 $(87 \%)$ reported carrying out surveillance tests for ovarian cancer, $35 / 76$ (46\%) for cervical cancer, 8/76 (11\%) for vulval cancer and $7 / 76(9 \%)$ for endometrial cancer. Patients were usually discharged after 5 years $(82 / 117(70 \%))$, whereas three $(3 \%)$ were discharged after 4 years, nine $(8 \%)$ after three years and one $(1 \%)$ after 2 years.

Conclusions: Practice varied but most used a standard hospital-based protocol of appointments for 5 years and routine tests were performed usually for women with ovarian cancer. A minority utilised nurse-led or telephone follow-up. General practitioners were rarely involved in routine care. A randomised study comparing various models of follow-up could be considered.

\section{INTRODUCTION}

Traditionally, patients who have had treatment for cancer are kept on regular review in

\section{ARTICLE SUMMARY}

Article focus

- Follow-up after treatment for cancer is a resource-intense area of clinical practice which does not have clear benefits for patients.

- Doctors and nurses involved in care for women with gynaecological cancer were invited to respond to a questionnaire survey.

- A survey is presented of current follow-up after treatment for gynaecological cancer in the UK.

Key messages

- There is a variation of follow-up practice throughout the UK.

- A minority used nurse-led or telephone follow-up as opposed to a conventional series of hospital outpatient appointments to see a doctor.

Strengths and limitations of this study

- This is the first study to report the extent of patient-initiated, specialist nurse or telephone follow-up for gynaecological cancer in the UK.

- Four UK cancer networks did not respond; there were variations of responses within networks and the response rate could not be calculated.

hospital outpatient clinics for a period between 5 and 10 years after completion of their treatment. ${ }^{1}$ The aims may be to detect recurrence of tumour, to monitor late effects of treatment, to collect data and to offer patients an opportunity to raise concerns or anxieties arising from their cancer. ${ }^{2-4}$ The assumption behind this approach is that early detection of recurrence will be of benefit to patients $^{5} 6$ and that monitoring side effects and anxieties will allow helpful interventions that will improve the quality of life.

Routine follow-up is a time-consuming and expensive process with many hundreds of patients attending clinics in each hospital every year at an NHS tariff of $£ 118$ per visit. ${ }^{7}$ In Wales alone, we estimate that the cost of this follow-up is in excess of $£ 1 \mathrm{~m}$ annually. If the object is the early detection of recurrent disease, studies investigating recurrence for 
breast cancer reported most recurrences presenting between scheduled clinic appointments. ${ }^{8-13}$ This is also seen with patients having had treatment for early stage endometrial and cervical cancers. ${ }^{14}{ }^{15}$ Gynaecological patients may wait for their next routine appointment to disclose their symptoms. ${ }^{16}$ There is no prospective randomised evidence to suggest that follow-up improves survival for ovarian cancer ${ }^{4}$ or for cervical cancer. ${ }^{17}$ Also, there is no evidence that intensive follow-up improves survival for endometrial cancer. ${ }^{14}{ }^{18-23}$ A cost-benefit analysis for endometrial carcinoma showed that one asymptomatic recurrence was detected in every 653 consultations. ${ }^{23}$ In a review of 12 studies, only $30 \%$ of endometrial cancer recurrences were asymptomatic and methods of follow-up were unrelated to survival. ${ }^{24}$ As around $20 \%$ of all female cancer survivors have had gynaecological malignancy, a cost-effective form of surveillance is important. ${ }^{25}$

In clinical practice, there appears to be no rationale available for any particular follow-up protocol. ${ }^{26}$ As there is a lack of any demonstrable survival benefit for the follow-up of gynaecological cancer patients, other schedules of care could be considered. Telephone consultations can free oncologists' clinic time and is convenient for patients. Follow-up may be in primary care, a hospitalbased nurse-led clinic, by telephone or at the request of the patient. Despite its place in standard healthcare, the benefits of routine follow-up following treatment for gynaecological cancer have received little scrutiny and have rarely been subjected to formal assessment.

In order to determine a baseline of current practice for gynaecological cancer follow-up in the UK, we have performed a survey of cancer follow-up in gynaecological oncology. The intention is to investigate who performs follow-up, for what duration and how this is achieved to see if there is a possibility to improve the quality of care offered to patients after their cancer treatment.

\section{MATERIALS AND METHODS}

The follow-up survey was designed using the Bristol Online Survey (BOS) to provide electronic data capture and data management support to the questionnaire. BOS is an easy-to-use survey tool which allows surveys to be developed, deployed and analysed via the internet. The data set was managed by the Information Systems Department at the North Wales Organisation of Randomised Trials (Bangor University). Data were anonymised and then exported to the databases held in SPSS (V.18.0; SPSS, Chicago, Illinois, USA). The e-survey targeted gynaecological cancer secondary care practitioners (incorporating surgical and non-surgical specialists) in all cancer networks in the UK. It was available online using an electronic web link from June to September 2012. An initial invitation email and a reminder with the web link were sent through the Principal Investigator (PI) distribution list to all 441 members of the British Gynaecological Cancer Society (BGCS) and all 71 members of the National Forum of Gynaecological Oncology Nurses (NFGON). A news release published in June 2012 on the BGCS website also invited members to take part in the survey. It is possible that respondents could provide multiple replies but no two responses from the same network were identical.

Investigators, in consultation with BGCS and a patient representative, designed the questionnaire which was organised around three themes (see questionnaire in the online supplementary appendix). The first comprised questions related to practice setting (ie, organisation and hospital) and respondent characteristics (ie, profession and medical specialty). The second comprised questions related to the use of standard protocols for follow-up. The bulk of the questionnaire addressed information about the different schedules of follow-up and which surveillance tests were used routinely in follow-up practices for different cancers. We listed four possible types of follow-up appointments: clinician-led (traditional), nurse-led, telephone follow-up and patientinitiated follow-up. Telephone follow-up appointments were defined as a prearrangement for a member of the cancer team to contact the patient by telephone without a need for the patient to attend hospital. Patient-initiated follow-up was defined as a practice where the patient is not followed up in secondary care but seen only if the patient requests or initiates a contact, for example, if they are worried about a suspicion of recurrent disease.

Most answers were recorded as a binary variable (yes/ no answer) with additional, open text box response options throughout the questionnaire for comments and alternative suggestions.

Data were collated and presented as numbers and a percentage of positive responses. For those questions composed of a subset of questions, the number of positive responses in the main question was used as the denominator for the subset. The geographical spread of responses was mapped by also calculating responses on a network basis, grouping all answers from respondents within their cancer networks. Any positive response within the group was accepted as a positive network response. Textual answers were categorised and counted.

\section{RESULTS}

\section{Sample size and respondent characteristics}

Responses were received from 118 experts in gynaecological cancer drawn from the membership of BGCS and NFGON. Because the survey was conducted online with the request to take part being distributed widely by email, it is impossible to state how many had the opportunity to take part but did not. Therefore, the response rate has not been calculated. Nonetheless, we received responses from $86 \%(24 / 28)$ of the cancer networks in England and all cancer networks in Scotland (three), Wales (two) and Northern Ireland (one). Each responding cancer network provided between 1 and 14 
responses. One response was received from a surgical oncologist based in Greece who was excluded from the study as the objective was to assess the current practice of follow-up after gynaecological cancer treatment in the UK. Of the 117 respondents included in the study, $71(61 \%)$ worked in a cancer centre, $32(27 \%)$ in a cancer unit and $12(10 \%)$ reported working in both. Eighty-eight $(75 \%)$ respondents specialised in surgical oncology. Fifteen (13\%) specialised in clinical oncology and six $(5 \%)$ in medical oncology. The majority of the respondents $(83(71 \%))$ were doctors while nurses constituted less than a third of the sample $(32(27 \%))$. Full results are presented in table 1 .

\section{Standard follow-up protocols}

All respondents, with the exception of one surgical oncologist $(116 / 117(99 \%))$, had a standard follow-up protocol after completion of treatment. However, all 30 networks providing responses had at least one respondent reporting having protocols for follow-up. The vast majority of respondents provided follow-up in secondary care, while only two respondents (from different English cancer networks) reported that visits to primary care were part of their follow-up routine.

Most of the respondents $(87 / 116(75 \%))$ reported using different follow-up protocols for different tumour sites (eg, cervix and ovary) and 35/116 (30\%) reported having different protocols for different tumour types

\begin{tabular}{|c|c|}
\hline & $\mathbf{N}(\%)$ \\
\hline \multicolumn{2}{|l|}{ Region } \\
\hline England & $102(87)$ \\
\hline Wales & $7(6)$ \\
\hline Scotland & $5(4)$ \\
\hline Northern Ireland & $3(3)$ \\
\hline \multicolumn{2}{|l|}{ Organisation } \\
\hline Cancer centre & $71(61)$ \\
\hline Cancer unit & $32(27)$ \\
\hline Cancer unit and cancer centre & $12(10)$ \\
\hline Other* & $2(2)$ \\
\hline \multicolumn{2}{|l|}{ Specialty } \\
\hline Surgical oncology & $73(62)$ \\
\hline Medical oncology & $6(5)$ \\
\hline Clinical oncology & $15(13)$ \\
\hline Surgical and medical oncology & $6(5)$ \\
\hline Surgical and clinical oncology & $1(1)$ \\
\hline Surgical, medical and clinical oncology & $8(7)$ \\
\hline Other† & $8(7)$ \\
\hline \multicolumn{2}{|l|}{ Profession } \\
\hline Medical & $83(71)$ \\
\hline Nursing & $32(27)$ \\
\hline Other & $2(2)$ \\
\hline \multicolumn{2}{|c|}{$\begin{array}{l}\text { *Gynaecology unit in a chemotherapy centre }(n=1) \text { and hospital } \\
(n=1) \text {. } \\
\text { †Clinical nurse specialist }(n=2) \text {, nursing }(n=2) \text {, gynaecology }(n=2) \text {, } \\
\text { gynaecology and surgical oncology }(n=1) \text { and pathology }(n=1) \text {. } \\
\text { †Consultant radiographer }(n=1) \text { and missing response }(n=1) \text {. }\end{array}$} \\
\hline
\end{tabular}

(eg, well or poorly differentiated). On a cancer network basis, different protocols for different tumour sites were reported from 29/30 (97\%) networks. Different protocols for different tumour types were reported by respondents from 17/30 (57\%) networks.

\section{Composition follow-up appointments}

All respondents in our sample reported that they followed up patients after completion of gynaecological cancer treatment. One hundred and fifteen $(98 \%)$ reported hospital scheduled regular follow-up appointments from which the patient could be discharged if she remained disease-free after a specified period of time. This follow-up was augmented or replaced by a telephone follow-up in 29 responses $(25 \%)$ and patient-initiated appointments in 38 responses $(32 \%)$. A combination of all three forms of follow-up was reported by 11 respondents (a total of 54 respondents offered more than one modality of follow-up). Of these, 18/54 (33\%) reported that patients have an opportunity to attend either a medical-led or a nurse-led clinic. However, 6/18 (33\%) did not have a protocol to allocate patients to each clinic. For patientinitiated appointments, 10/38 (26\%) did not have a protocol with contact details (eg, a secretary, Macmillan nurse or general practitioner) for self-referrals. A total of 80/117 $(68 \%)$ cancer specialists from $27 / 30$ networks $(90 \%)$ also offered combined follow-up clinics with other specialties (eg, combined surgical and medical oncology or surgical and clinical oncology clinics).

Virtually all respondents reported in the case of sudden events that symptomatic patients could arrange an appointment in less than 2 weeks. Seven (6\%) respondents from five different cancer networks answered that their practices scheduled urgent appointments in a period of 2-4 weeks, from which two of them also scheduled urgent patient-initiated appointments in the same time frame.

Follow-up in hospital was mainly performed by doctors (67\% for scheduled regular appointments and $63 \%$ for patient-initiated appointments), while telephone followup care was provided in its majority by nurses $(76 \%)$. Full details are illustrated in table 2.

\section{Duration of follow-up and surveillance tests}

The survey asked respondents whether they performed any type of routine surveillance test during follow-up. Routine tests were requested by $65 \%(76 / 117)$ respondents, of which $87 \%(66 / 76)$ requested tests for ovarian cancer, $46 \%(35 / 76)$ for cervical cancer, $11 \%(8 / 76)$ for vulva cancer and 9\% (7/76) for endometrial cancer. In addition, respondents were asked to provide details of when these tests were performed but only a few responses were obtained. Table 3 shows the distribution of the different type of tests employed during follow-up.

CA125 measurement was the most frequently used test $(60 / 66(91 \%))$ during the follow-up of ovarian cancer patients. Other blood tests $(8 / 66(12 \%))$, for example, $\alpha$-fetoprotein, carcinoembryonic antigen, CA19.9 and 
Table 2 Type and frequency of occurrence of differing modes of follow-up

\begin{tabular}{|c|c|c|c|}
\hline & Regular & Telephone & Patient initiated \\
\hline Positive responses & $115 / 117(98 \%)$ & $29 / 117(25 \%)$ & $38 / 117(32 \%)$ \\
\hline \multicolumn{4}{|c|}{ Urgent follow-up bookings (weeks) } \\
\hline$<2$ & $108 / 115(94 \%)$ & $29 / 29(100 \%)$ & $35 / 38(92 \%)$ \\
\hline $2-4$ & $7 / 115(6 \%)$ & $0(0 \%)$ & $2 / 38(5 \%)$ \\
\hline \multicolumn{4}{|l|}{ Responsible for follow-up } \\
\hline Doctors & $77 / 115(67 \%)$ & $4 / 29(14 \%)$ & $24 / 38(63 \%)$ \\
\hline Nurses & $0(0 \%)$ & $22 / 29(76 \%)$ & $2 / 38(5 \%)$ \\
\hline Doctors and nurses & $36 / 115(31 \%)$ & $3 / 29(10 \%)$ & $11 / 38(29 \%)$ \\
\hline Other* & $2 / 115(2 \%)$ & $1 / 29(3 \%)$ & $1 / 38(3 \%)$ \\
\hline
\end{tabular}

inihibin were also requested. The routine use of CT and MRI scans for ovarian cancer was reported by $9 / 66$ (14\%) respondents (one respondent used both CT and MRI). Eight respondents stated that the CA125 test was performed at each visit. Another seven reported that CA125 was performed every 3 months during the first year after completion of treatment, of which two reported that they carried on with routine testing during the second year and four up to the fifth year every 6 months.

The use of MRI (15/35 (43\%)) was the most common investigation employed in the follow-up of cervical cancer with cervical or vaginal cytology being the second most common method (14/35 (40\%)). A wide variety of tests $(8 / 35(23 \%))$ were reported in the follow-up of this type of cancer. Two of the respondents stated that they performed vault cytology for cervical cancer patients annually over a period of 5 years following hysterectomy, while three specialists reported carrying out the test at 6 and 18 months post-treatment.

Most respondents discharged their patients after 5 years of follow-up $(82 / 117(70 \%))$, three $(3 \%)$ after 4 years, nine $(8 \%)$ after three years and one $(1 \%)$ after 2 years, whereas $22 / 117(19 \%)$ respondents did not answer this question.

\section{DISCUSSION}

The current survey is the first evidence reporting the extent of patient-initiated, specialist nurse or telephone follow-up for gynaecological cancer in the UK. There were no respondents for four cancer networks and because the survey was online, a response rate could not be calculated. While we know the membership of the professional societies invited to respond, we do not know whether the entire membership received or read their invitations to participate in our survey. Nonetheless, a low-response rate could introduce a potential source of bias if the answers from respondents were not representative of their relevant professional communities. Different protocols for different tumour sites and types and the use of combined specialty follow-up clinics were reported more often from network responses than for individual responses because positive network responses included respondents with at least one positive response in each network. Unfortunately, we could not calculate the agreement level within networks because of the small numbers of respondents from each network. The lack of consistency of responses within networks is again a potential source of error as such responses may not accurately reflect local practice. We did not review the content of the follow-up protocols, and so we cannot verify if these variations represent locally approved practice within each network. Network guidelines may be adapted for local use or not followed exactly, so variations within networks could be expected. Our survey shows that all gynaecological cancer networks providing responses have protocols for follow-up after treatment. Follow-up for patients treated for gynaecological cancer is mainly performed by

Table 3 Frequency of surveillance tests reported by type of test and cancer type

\begin{tabular}{lllllrrrr}
\hline & Ultrasoundt & CA125 & Other blood tests & CT & MRI & Cytology & Other & Total \\
\hline Ovarian & 5 & 60 & 8 & 5 & 4 & 0 & 0 \\
Cervical & 1 & 0 & 4 & 0 & 15 & 14 & 82 \\
Endometrial & 1 & 1 & 0 & 0 & 1 & 1 & 3 \\
Vulval & 0 & 0 & 0 & 0 & 1 & 0 & 0 \\
\hline
\end{tabular}

${ }^{*}$ Other blood tests includes: other tumour markers $(n=8)$ for ovarian cancer and squamous cancer antigen $(n=3)$

for cervical cancer.

†Ultrasound includes: abdominal and transvaginal ultrasound.

¥Other includes: colposcopy $(n=2)$ for cervical cancer and vulvoscopy $(n=3)$ for vulval cancer.

CA125, cancer antigen 125. 
doctors in secondary care. Patient-initiated follow-up was offered by $32 \%$ of respondents and telephone follow-up was offered by $25 \%$. A large minority of patient-initiated follow-up and combined follow-up schedules did not have protocols to guide practice. The most common duration of routine follow-up was for 5 years. Few routine tests are undertaken during follow-up to detect recurrence and they show no consistency, particularly for cervical cancer. Of the 35 respondents who requested tests for cervical cancer follow-up, $15(43 \%)$ requested MRI and $14(40 \%)$ requested cytology. However, cervical cytology is recommended for the follow-up of early-stage cervical cancer if the cervix is conserved and is based on expert opinion rather than on evidence. ${ }^{27}$ Variation in the routine use of tests during follow-up is not surprising with the lack of evidence to guide clinical management. The exception is CA125 testing following treatment for ovarian cancer where $52 \%$ of all respondents recommended CA125 monitoring despite grade one evidence to demonstrate that routine CA125 measurements do not provide a survival benefit with early treatment of relapse. ${ }^{28}$ Furthermore, there appears to have been no recent change of this practice in our survey, as monitoring with CA125 testing was reported in 24 of the 34 UK networks $(71 \%)$, whereas $67 \%$ of networks recommended such testing in an earlier survey by Kew and Cruickshank. ${ }^{1}$ Respondents were asked not to include tests requested during treatment, but tests could have been included as part of the trial protocols. Vistad et at also published the results of a web-based survey of practice among gynaecological oncologists across Europe and reported that $47 \%$ of the 375 respondents considered follow-up with general practitioners to be acceptable. Other options for care were not considered and the response rate was thought to be below $20 \%$.

From other research, patients with previous ovarian cancer rated CA125 testing as the most important part of follow-up. ${ }^{29}$ Furthermore, knowledge of recurrence, whether treatable or not, appears to be useful to patients ${ }^{30}$ and information should be provided to detail the scope and limitations of follow-up. ${ }^{3}$ Rapid access to oncological assessment at recurrence may be more important than offering frequent routine appointments. ${ }^{1431}$ Knowing that different schedules of follow-up do not have an impact upon survival, delegation of routine follow-up could be to other carers. ${ }^{6}$ Follow-up may be in primary care, a hospital-based nurse-led clinic, by telephone or at the request of the patient. An individualised approach to follow-up is likely to be important to concentrate care for those perceived to be at a greater risk of recurrent disease or other issues of survivorship. This may include risk stratification, where there are effective interventions for physical, psychological and social issues, as well as needs assessments, which are clearly patient-centred, as defined by the National Cancer Survivorship Initiative. ${ }^{32}$ Follow-up has to be multidisciplinary, designed for detection of morbidity as well as recurrence and with good communication between professional groups. Informed patient choice regarding the mode and frequency of follow-up is important. Reducing the frequency of follow-up appointments may not place an increased demand on unnecessary patient-initiated extra hospital appointments and patients may prefer fewer appointments. ${ }^{31}$ The ideal time of advising a patient about a preferred form of follow-up is unclear but may be shortly after all modalities of treatment have been completed.

Healthcare providers should be informed by prospective data on the validity of alternative strategies for gynaecological cancer follow-up, which is already a minority part of current UK practice. The North Wales Organisation for Randomised Trials in Health, in collaboration with several leading gynaecological oncologists, has previously developed a proposal for a randomised study to assess the value of hospital follow-up of endometrial cancer (Follow-up in Gynaecological Cancer Units: randomised controlled trial (RCT) for Endometrium or FIGURE). The proposal was for a multicentre randomised trial comparing standard (hospital) follow-up with a patient-initiated review. The endpoints were to be quality of life and survival with a planned recruitment of 2200 patients to detect an effect size of 0.2 . Unfortunately, although the proposal was well received, it was impossible to agree on the level of funding to allow the study to proceed. The Endometrial Cancer Telephone Follow-up Trial (ENDCAT) ${ }^{33}$ has started recently. This is a study comparing traditional hospital follow-up with telephone follow-up by specialist gynaecology oncology nurses with primary outcomes of psychological morbidity and patient satisfaction. Again, this is a randomised trial for endometrial cancers only and is at a single centre. It is currently recruiting to schedule and is due to close in 2014. A Trial between Two Follow-up Regimens with Different Intensity in Endometrial Cancer-Treated Patients (TOTEM) ${ }^{34}$ is a further ongoing but multicentre randomised trial in Italy, comparing overall survival, progression-free survival, complications, proportion of asymptomatic relapse and the proportion of patients completing each regimen for follow-up. It is due to close in 2015.

Our study has demonstrated that the vast majority of follow-up still reflects traditional patterns, with only about a third of the practitioners incorporating more flexible follow-up routines. However, the evidence base for changing practice to a less intensive follow-up programme for gynaecological cancer is poor and Vistad et al ${ }^{15}$ reported no randomised studies on this subject. A trial similar to an early version of FIGURE should be revisited, which included follow-up for more than one gynaecological cancer site. In the present constrained financial environment, to continue to use patterns of follow-up for gynaecological cancers which are neither evidence-based nor affordable is inappropriate. A multicentre RCT could assess the clinical benefits and costs of routine hospital follow-up in comparison with the patient being empowered to choose her preferred format of follow-up for 
most gynaecological cancers. The current survey may inform design of such a trial by providing data from the UK concerning national practice.

Acknowledgements The authors would like to thank Dr David Hunnisett, IS Manager, North Wales Organisation for Randomised Trials in Health (\& Social Care), Institute of Medical \& Social Care Research (IMSCaR), Bangor University, Y Wern, George Site, Holyhead Road, Bangor, Gwynedd LL57 2PZ.

Contributors RW conceived the idea for the survey and all authors designed the questionnaire and the method of the survey. RW, YS, NS and SL interpreted the data. All authors provided the text and reviewed the draft script and SL provided modifications as requested. All authors critically reviewed the script modifications.

Funding This research received no specific grant from any funding agency in the public, commercial or not-for-profit sectors.

Competing interests None.

Provenance and peer review Not commissioned; externally peer reviewed.

Data sharing statement Extra data can be accessed via the Dryad data repository at http://datadryad.org/ with the doi:10.5061/dryad.h7234.

Open Access This is an Open Access article distributed in accordance with the Creative Commons Attribution Non Commercial (CC BY-NC 3.0) license, which permits others to distribute, remix, adapt, build upon this work noncommercially, and license their derivative works on different terms, provided the original work is properly cited and the use is non-commercial. See: http:// creativecommons.org/licenses/by-nc/3.0/

\section{REFERENCES}

1. Kew FM, Cruickshank DJ. Routine follow-up after treatment for a gynaecological cancer: a survey of practice. Int J Gynecol Cancer 2006;16:380-4.

2. Kerr-Wilson RH, McCrum A. Follow-up of patients with gynaecological cancer. Aust NZ J Obstet Gynaecol 1995;35:298-9.

3. Kew FM, Galaal K, Manderville H, et al. Professionals' and patients' views of routine follow-up: a questionnaire survey. Int J Gynecol Cancer 2007; 17:557-60.

4. Kew F, Galaal K, Bryant A, et al. Evaluation of follow-up strategies for patients with epithelial ovarian cancer following completion of primary treatment. Cochrane Database Syst Rev 2011;4:CD006119. doi: 10.1002/14651858.CD006119.pub2 (accessed 16 Apr 2013).

5. Lajer $\mathrm{H}$, Elnergaard S, Christensen RD, et al. Survival after stage IA endometrial cancer; can follow-up be altered? A prospective nationwide Danish survey. Acta Obstet Gynecol Scand 2012;91:976-82.

6. Vistad I, Cvancarova M, Salvesen HB. Follow-up of gynecological cancer patients after treatment-the views of European experts in gynecologic oncology. Acta Obstet Gynecol Scand 2012;91:1286-92.

7. Department of Health. 2011. NHS reference Costs 2010-11. http:// dh.gov.uk.en/Publicationsandstatistics/Publications/Publications PolicyAndGuidance/DH_131140 (accessed 17 Dec 2012).

8. Brøyn T, Frøyen J. Evaluation of routine follow-up after surgery for breast carcinoma. Acta Chir Scand 1982;148:401-4.

9. Grunfeld E, Yudkin P, Adewuyl-Dalton R, et al. Routine follow up of breast cancer in primary care: randomised trial. BMJ 1996;313:665-9.

10. Pandya KJ, McFadden ET, Kalish LA, et al. A retrospective study of earliest indicators of recurrence in patients on Eastern Cooperative Oncology Group adjuvant chemotherapy trials for breast cancer. A preliminary report. Cancer 1985;55:202-5.

11. Scanlon EF, Oviedo MA, Cunningham MP, et al. Preoperative and follow-up procedures on patients with breast cancer. Cancer 1980;46(4 Suppl):977-9.

12. Winchester DP, Sener SF, Khandekar JD, et al. Symptomatology as an indicator of recurrent or metastatic breast cancer. Cancer 1979;43:956-60.
13. Zwaveling A, Albers GH, Felthuis W, et al. An evaluation of routine follow-up for detection of breast cancer recurrences. J Surg Oncol 1987;34:194-7.

14. Shumsky AG, Stuart GC, Brasher PM, et al. An evaluation of routine follow-up of patients treated for endometrial carcinoma. Gynecol Oncol 1994;55:229-33.

15. Vistad I, Moy BW, Salvesen HB, et al. Follow-up routines in gynecological cancer-time for a change? Acta Obstet Gynecol Scand 2011:90:707-18.

16. Olaitan A, Murdoch J, Anderson R, et al. A critical evaluation of current protocols for the follow-up of women treated for gynecological malignancies: a pilot study. Int J Gynecol Cancer 2001;11:349-53.

17. Elit L, Fyles AW, Devries MC, et al. Gynecology Cancer Disease Site Group. Follow-up for women after treatment for cervical cancer: a systematic review. Gynecol Oncol 2009;114:528-35.

18. Agboola O, Grunfeld E, Coyle D, et al. Costs and benefits of routine follow-up after curative treatment for endometrial cancer. Can Med Assoc J 1997;157:879-86.

19. Allsop JR, Preston J, Crocker S. Is there any value in the long-term follow-up of women treated for endometrial cancer? Br J Obstet Gynaecol 1997;104:122.

20. Gadducci A, Cosio S, Fanucchi A, et al. An intensive follow-up does not change survival of patients with clinical stage I endometrial cancer. Anticancer Res 2000;20:1977-84.

21. Owen $P$, Duncan ID. Is there any value in the long-term follow-up of women treated for endometrial cancer? Br J Obstet Gynecol 1996;103:710-13.

22. Reddoch JM, Burke TW, Morris M, et al. Surveillance for recurrent endometrial carcinoma: development of a follow-up scheme. Gynecol Oncol 1995;59:221-5.

23. Salvesen HB, Akslen LA, Iversen $\mathrm{T}$, et al. Recurrence of endometria carcinoma and the value of follow-up. Br J Obstet Gynaecol 1997;104:1302-7.

24. Fung-Kee-Fung M, Dodge J, Elit L, et al. Follow-up after primary therapy for endometrial cancer: a systematic review. Gynecol Oncol 2006;101:520-9.

25. Salani R, Backes FJ, Fung MF, et al. Posttreatment surveillance and diagnosis of recurrence in women with gynecologic malignancies: Society of Gynecologic Oncologist recommendations. Am J Obstet Gynecol 2011;204:466-78.

26. Sartori E, Pasinetti B, Chiudinelli F, et al. Surveillance procedures for patients treated for endometrial cancer: a review of the literature. Int $J$ Gynecol Cancer 2010;20:958-92.

27. Luesley D, Leeson S. eds. Colposcopy and programme management. Guidelines for the NHS cervical screening programme. 2nd edn. NHSCSP publication no. 20, 2010

28. Rustin GJ, van der Burg ME, Griffin CL, et al. MRC OV05; EORTC 55955 investigators. Early versus delayed treatment of relapsed ovarian cancer (MRC OV05/EORTC 55955): a randomised trial. Lancet 2010;376:1155-63.

29. Kew FM, Galaal K, Manderville $H$. Patients' views of follow-up after treatment for gynaecological cancer. J Obstet Gynaeco 2009;29:135-42.

30. Papagrigoriadis S, Heyman B. Patients' views on follow up of colorectal cancer: implications for risk communication and decision making. Postgrad Med J 2003;79:403-7.

31. Gulliford T, Opomu M, Wilson E, et al. Popularity of less frequent follow up for breast cancer in randomised study: initial findings from the hotline study. BMJ 1997;314:174-7.

32. Watson EK, Rose PW, Neal RD, et al. Personalised cance follow-up: risk stratification, needs assessment or both? $\mathrm{Br} J$ Cancer 2012;106:1-5.

33. ENDCAT: Comparing hospital and telephone follow up for women treated for endometrial cancer: Endometrial cancer telephone follow up trial. ISRCTN75220876. doi: 10.1186/ISRCTN75220876 (accessed 31 Jan 2012).

34. TOTEM: Trial between two follow up regimens with different test intensity in endometrial cancer treated patients. NCT00916708. http://www.cancer.gov/clinicaltrials/search/view?cdrid=647441 \&version=HealthProfessional\&protocolsearchid=8392570 (accessed 31 Jan 2012) 